\title{
Design Method of Dual Phase Hy-Vo Silent Chain Transmission System
}

\author{
Yabing Cheng* - Yang Wang - Lei Li - Shuaibing Yin - Lichi An - Xiaopeng Wang \\ Jilin University, School of Mechanical Science and Engineering, China
}

Based on the theory that the Hy-Vo silent chain meshes with the sprocket, and the related condition that the Hy-Vo silent chain sprocket meshes with a tool, multi-variations of the Hy-Vo silent chain and sprocket are designed. The meshing design system of the dual phase Hy-Vo silent chain plate-sprocket-tool is built. The design method of the dual phase Hy-Vo silent chain transmission system is presented. Combined with a concrete example, the dual phase Hy-Vo silent chain transmission system model is established, and the dual phase transmission system is decomposed into two single-phase transmission systems. The research shows that the instantaneous transmission ratio of a dual phase transmission system may be different from the instantaneous transmission ratio of a decomposed single-phase transmission system. If the two pitches of the dual phase sprocket are equal and the two groups of pressure angles are the same, the largest fluctuation quantity of the dual phase transmission system is less in comparison with the single-phase transmission system, and the polygon effect is smaller. The design method will provide a corresponding theoretical guidance for the design of other multi-phase chain systems.

Key words: dual phase transmission, Hy-Vo silent chain, multi-variation, fluctuation quantity, polygon effect

Highlights

- $\quad$ Designed the multi-variation Hy-Vo silent chain and sprocket.

- Established the design system of the dual phase Hy-Vo silent chain plate-sprocket-tool.

- $\quad$ Studied the polygon effect through mathematical analysis and simulation analysis.

- $\quad$ Reduced the largest fluctuation quantity and the polygon effect of drive system.

- $\quad$ Provided the theoretical guidance for the design of multi-phase chain system.

\section{O INTRODUCTION}

The Hy-Vo silent chain is a series of high-end products in the field of mechanical transmission. In recent years, with the continuous development of highspeed chain transmission technology and the growth of different personalized needs regarding automotive engines, the Hy-Vo silent chain and sprocket have undergone a continuous variation development trend; this trend represents significant change and innovation for Hy-Vo silent chain transmission technology.

Research on Hy-Vo silent chain transmission systems has a long history. Meng et al. proposed the proper meshing conditions for the Hy-Vo silent chain and the sprocket [1], and the meshing system of the new silent chain plate-sprocket-hob was built; meanwhile, the design system of Hy-Vo silent chain transmission system was established [2] to [3], and the wear failure mechanism was explored through the road experiments [4] to [5]. Wang et al. designed a new sprocket tooth profile to reduce the polygonal action and meshing impact. If this method were adopted, the meshing impact and friction of the chain would be decreased [6]. Liu et al. changed the plate with a nano-structured metal mesh-polyurethane composite material, and proposed a new type of double pitch silent chain and a new silent chain assembly method
[7] to [8]. Sun et al. obtained the ANSI sprocket tooth profile and involute sprocket tooth profile, and studied the effects of the dynamic tension of the chain in the silent chain drive [9]. Bucknor et al. developed a quasistatic model for a silent chain drive and modelled the kinematic effects of rocker-pin clearances [10]. Troedsson and Vedmar [11] and Stephenson et al. [12] researched the oscillations and forces of the chain drive; the model has been established to work at moderate and high speed conditions. Schwab et al. presented [13] a procedure to estimate the maximum contact force of the impact model, and Flores et al. [14] presented a general methodology to constrain rigid multibody systems. Based on these findings, Pereira et al. [15] built chain drive automatic multibody models from a minimal set of data, and aimed to overcome the difficulty of building manually complex models of chain drives.

At present, the multi-variation design on $\mathrm{Hy}-\mathrm{Vo}$ silent chain and sprocket includes meshing mechanism variation, shape variation and parameter variation. The change process for meshing mechanism variation is outer meshing, inner-outer compound meshing and outer meshing with inner-outer compound meshing. "Shape variation" refers to major parts structure variations of Hy-Vo silent chains, such as plate hole shape variation, shape variation about pin 
cross section, guide plate shape variation. "Parameter variation" refers to the main parameter variations of the Hy-Vo silent chain and sprocket, including the positioning offset angle variation, the distance variation of benchmark circle centre, apothem variation, big side surface curvature radius variation of rocker-pin, the variation of plate tooth profile angle, the variation of sprocket pressure angle, and the variation of tool normal tooth angle.

It should be noted that regardless of what the abovementioned variation is, they are all variations in the single-phase transmission system. In this paper, the variation of the transmission system is studied based on the single-phase Hy-Vo silent chain transmission system, the dual phase Hy-Vo silent chain transmission system is designed, and the multivariation design method of the dual phase Hy-Vo silent chain plate and sprocket are proposed, and the fluctuation quantity of the dual phase Hy-Vo silent chain transmission system is researched.

\section{THE MESHING DESIGN OF THE DUAL PHASE Hy-VO SILENT CHAIN TRANSMISSION SYSTEM}

\subsection{The Parameter Design of the Dual Phase Hy-Vo Silent Chain Plate}

Supposing that $A$ is the hole pitch, $P$ is the basic pitch. $r$ is the big side surface curvature radius of the rockerpin. $S_{m}$ is the distance between the circle centre $O$ of the chain plate hole and the big side surface of the rocker-pin. $\gamma$ is the positioning offset angle when the rocker-pin is locked in the chain plate. $\alpha$ is the tooth profile angle of the Hy-Vo silent chain plate. $f$ is the benchmark apothem between the circle centre $O$ and chain plate outside linear profile. Fig. 1 shows the schematic diagram of the dual phase Hy-Vo silent a)

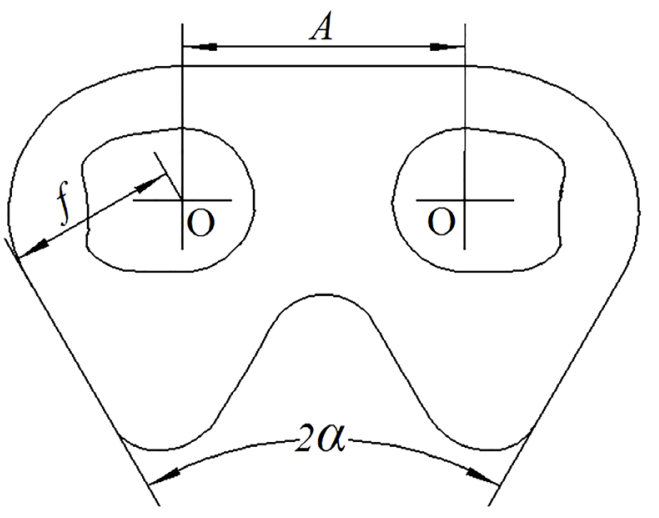

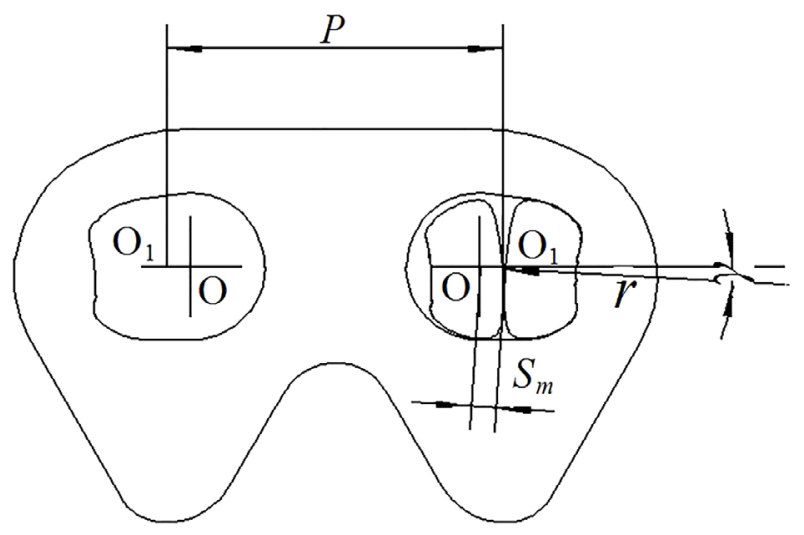

b)

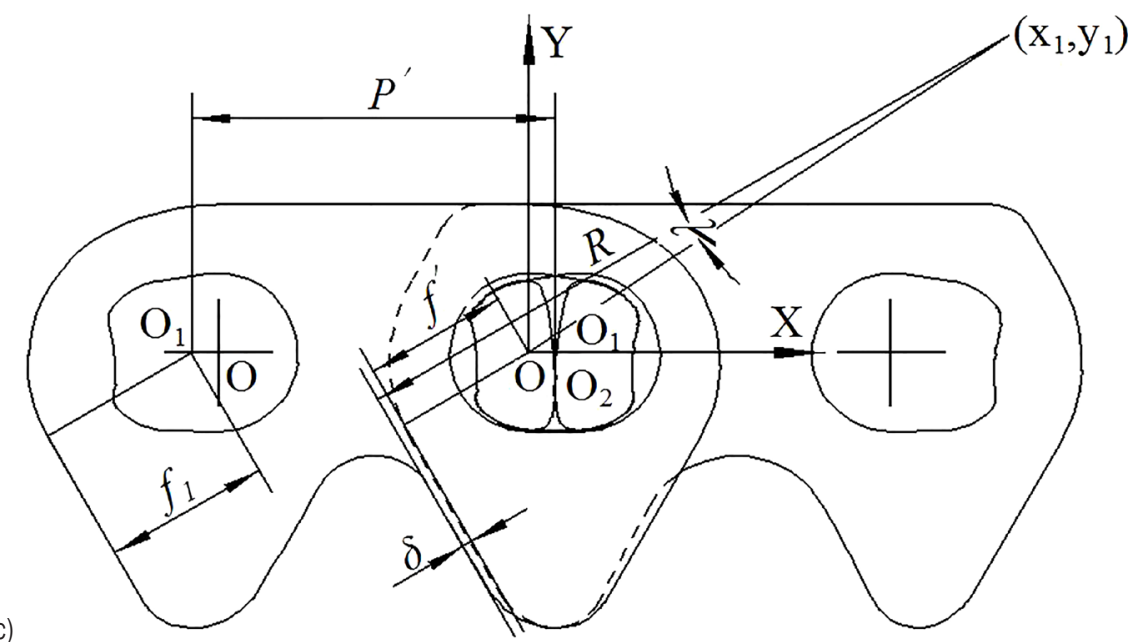

Fig. 1. The schematic diagram of the dual phase Hy-Vo silent chain in straightening 
chain in straightening. The coordinate system xoy is established on the circle centre $O . O_{1}$ is the extension intersection of the adjacent benchmark circle centre lines. $\mathrm{O}_{2}$ is the contact point of two rocker-pin at the action of tension.

Defining the distance of $O_{1}-O_{1}$ as the pitch of the dual phase Hy-Vo silent chain, in straightening:

$$
P^{\prime}=P=A+2\left[\left(r+S_{m}\right) \cos \gamma-r\right]
$$

where $f_{1}$ is the equivalent apothem between the point $O_{1}$ and chain plate outside linear profile:

$$
f_{1}=f-\left[\left(r+S_{m}\right) \cos \gamma-r\right] \cos \alpha .
$$

The inside profile overhang about the internal meshing plate is $\delta$, and the tooth profile curvature is $R$. A straight line goes through the inside tooth profile curvature centre and the circle centre $O$, and another straight line is not only perpendicular to chain plate outside linear profile but also passes through the inside tooth profile curvature centre, and the angle between the two straight lines is $\lambda$. The coordinates of the inside tooth profile curvature centre is as follows:

$$
\left\{\begin{array}{l}
x_{1}=\frac{\left(R-f^{\prime}-\delta\right) \sin \left(\lambda+60^{\circ}\right)}{\cos \lambda} \\
y_{1}=\frac{\left(R-f^{\prime}-\delta\right) \cos \left(\lambda+60^{\circ}\right)}{\cos \lambda}
\end{array},\right.
$$

where $f^{\prime}=f-\left(p^{\prime}-A\right) \cos \alpha$.

The load is transferred through the rolling role of the two rocker-pins in the dual phase Hy-Vo silent chain transmission system. When the adjacent links rotate mutually, the contact point in the big side surface of the rocker-pin will gradually move up, as shown in Fig. 2. The $h_{y}$ is the distance between the point $O_{1}$ and the point $O_{2}$. When the relative angle of the adjacent links is $2 \theta$ :

$$
\begin{aligned}
h_{y} & =\left(S_{m}+r\right) \sin (\gamma-\theta)+ \\
& +\tan \theta\left[\left(S_{m}+r\right) \cos (\gamma-\theta)-r\right] .
\end{aligned}
$$

When $h_{y}>0$, the point $O_{1}$ is below the point $O_{2}$. When $h_{y}=0$, the point $O_{1}$ coincides with the point $O_{2}$. When $h_{y}<0$, the point $O_{1}$ is above the point $O_{2}$.

At this moment, the working chain plate pitch is:

$$
P^{\prime \prime}=A+2\left[\frac{r+S_{m} \cos (\theta-\gamma)-r}{\cos \theta}\right] .
$$

Equivalent apothem $f_{1}$ is changed to:

$$
f_{2}=f-\left[\frac{\left(r+S_{m}\right) \cos (\theta-\gamma)-r}{\cos \theta}\right] \cos \alpha .
$$

\subsection{The Design System of the Dual Phase Hy-Vo Silent Chain Plate-Sprocket-Tool}

The dual phase sprocket structure (with 35 teeth, for example) is shown in Fig. 3. Because the dual phase sprocket teeth have a phase difference, each phase tooth may have a difference in pitch, module, pressure angle parameters, and the tools processing each phase tooth are different. Supposing the dual phase sprocket pitches are $P_{11}$ and $P_{12}$, the modules are $m_{11}$ and $m_{12}$, and the pressure angles of sprockets are $\alpha_{11}$ and $\alpha_{12}$.

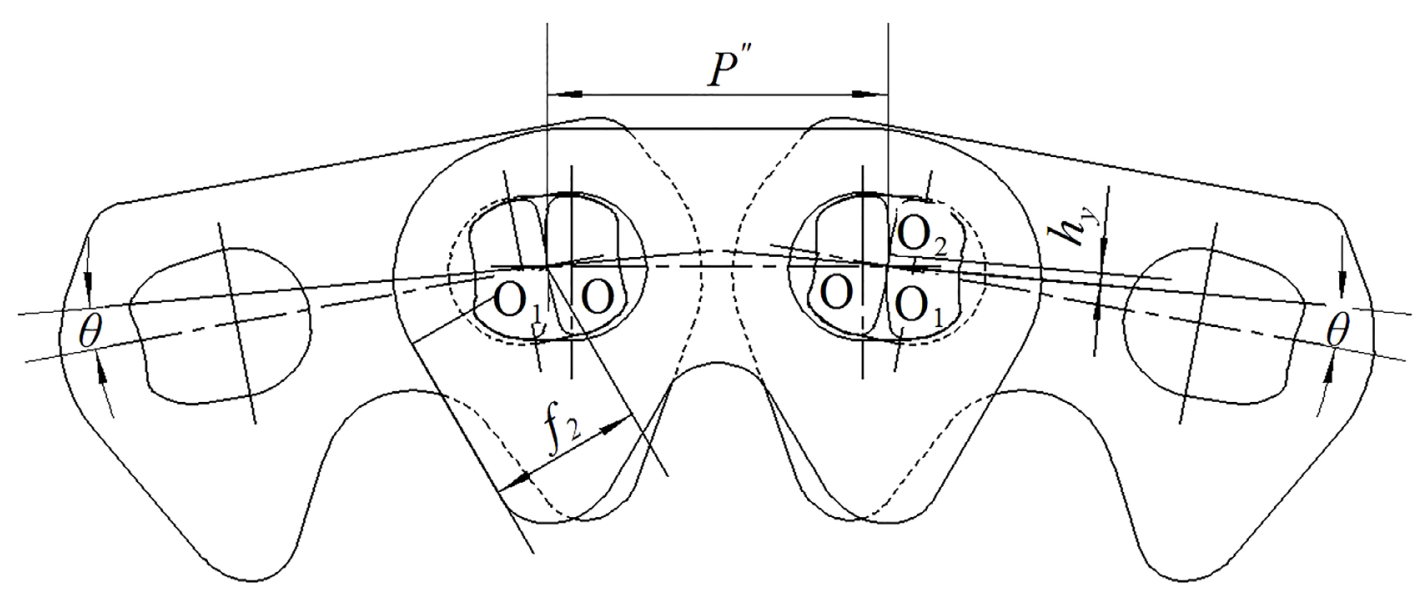

Fig. 2. The rotation schematic of dual phase Hy-Vo silent chain 


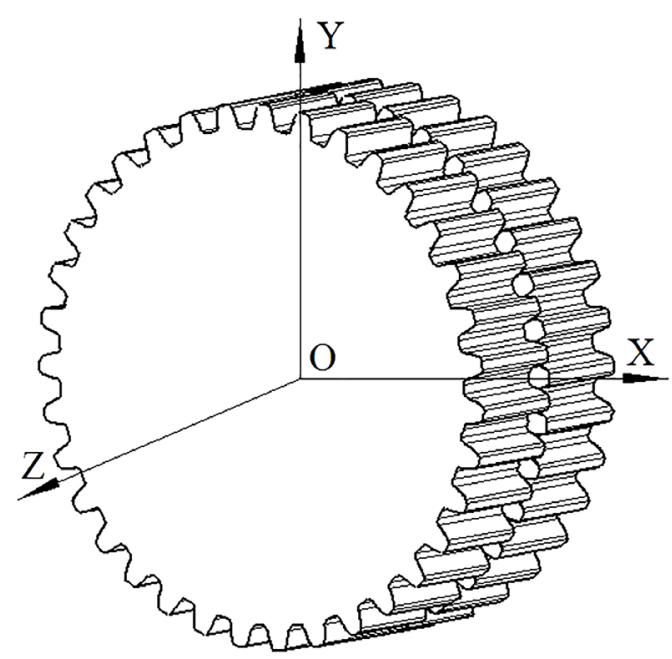

Fig. 3. The schematic diagram of the dual phase sprocket

The dual phase sprocket is projected onto xoy, as shown in Fig. 4. The solid line represents phase teeth whose sprocket tooth pitch is $P_{11}$, and the dotted line represents phase teeth that sprocket tooth pitch is $P_{12}$, and the phase difference of the sprocket tooth is $\sigma$. In the process of the chain plates are meshing with the dual phase sprocket, the working chain plate is regarded as a tool of the involute tooth. When the HyVo silent chain plates are surrounding the dual phase transmission sprocket in the proper meshing place, the twining round is formed, and the twining round diameter is $d_{c}$. The tooth slot symmetric centreline is $n-n$. The tooth slot symmetric centreline is intersected with the twining round one point $O_{1}$. The first tool normal tooth angle is $\alpha_{21}$, the first tool normal pitch is $P_{21}$, and the first tool module is $m_{21}$. According to conjugate meshing condition $P_{11} \cos \alpha_{11}=P_{21} \cos \alpha_{21}$ and making $\alpha_{21}=\alpha$, the first design system of the dual phase Hy-Vo silent chain plate-sprocket-tool is established. The dual phase sprocket centre is regarded as coordinate origin, the symmetrical centreline of the dual phase sprocket tooth is regarded as the vertical axis, the horizontal line that is through the centre of sprocket and vertical to the symmetrical centreline of the dual phase sprocket tooth is regarded as the horizontal axis, and the coordinate systems is established.

Supposing the Hy-Vo silent chain initial pitch is $P_{01}\left(P_{01}=P_{21}\right)$ and the initial apothem is $f_{01}$, the meshing between the tool $\left(P_{21}, \alpha_{21}\right)$ and the sprocket $\left(P_{11}, \alpha_{11}\right)$ could be regarded as the meshing between the Hy-Vo silent chain $\left(P_{01}, \alpha f_{01}\right)$ and the sprocket $\left(P_{11}, \alpha_{11}\right)$. If the dual phase sprocket tooth number is $Z$, and the relative rotating angle of the adjacent working plates is $2 \theta=2 \pi / z$.

The initial apothem $f_{01}$ is:

$$
f_{01}=f_{2}-\left(\cot \frac{\pi}{z}-\sqrt{3}\right)\left(P^{\prime \prime}-P_{21}\right) / 4 \text {. }
$$

The modification coefficient $x$ corresponds to the tool $\left(P_{21}, \alpha_{21}\right)$ is:

$$
x=\frac{\pi \cot \alpha_{21}}{4}-\frac{z}{2}+\frac{\pi}{2 \tan (\pi / z)}-\frac{\pi f_{01}}{P_{21} \sin \alpha_{21}} .
$$

When the outside linear profile of working link is fully meshed and located, providing $A C$ is the involute tooth profile tangent line, the sprocket base circle radius is $r_{b}$, and the involute tooth profile is:

$$
\Gamma\left\{\begin{array}{l}
x=r_{b}(\sin (\varnothing+\theta)-\varnothing \cos (\varnothing+\theta)) \\
y=r_{b}(\cos (\varnothing+\theta)+\varnothing \sin (\varnothing+\theta))
\end{array} .\right.
$$

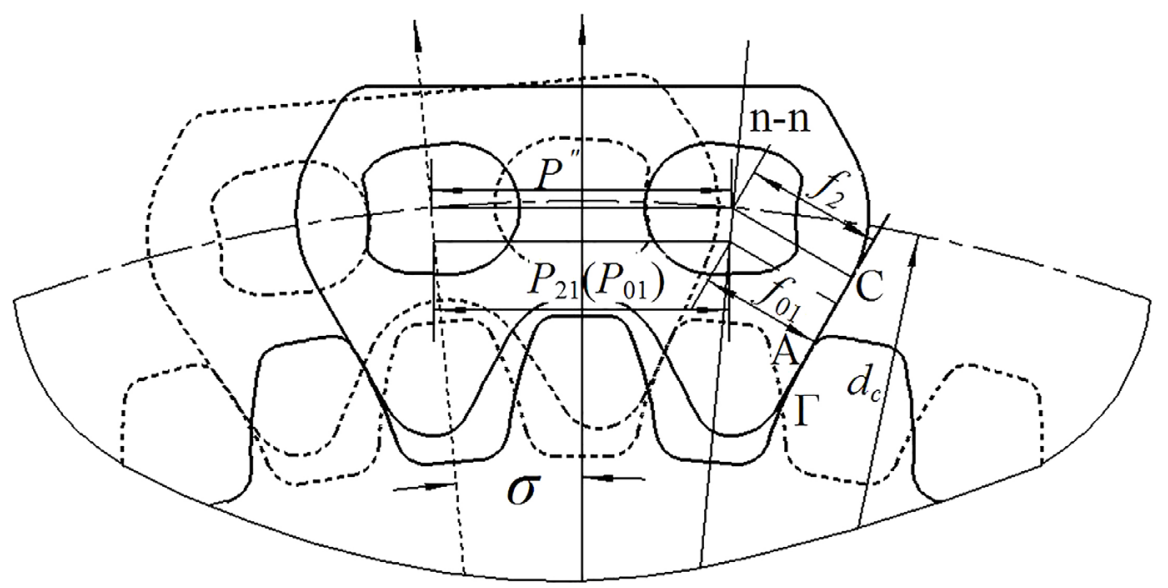

Fig. 4. The design system of the dual phase Hy-Vo silent chain plate- sprocket-tool 
The coordinate of the point $C$ is:

$$
\left\{\begin{array}{c}
x_{C}=\frac{P^{\prime \prime}}{2}+f_{2} \cos \alpha \\
y_{C}=\frac{P^{\prime \prime}}{2} / \tan \frac{\pi}{z}-f_{2} \sin \alpha
\end{array}\right.
$$

the coordinate of the tangent point $A$ is:

$$
\left\{\begin{array}{l}
x_{A}=r_{b} \sin \alpha-r_{b} \varnothing_{A} \cos \alpha \\
y_{A}=r_{b} \cos \alpha+r_{b} \varnothing_{A} \sin \alpha
\end{array}\right.
$$

in which, $\varnothing_{A}=\frac{y_{C} \sin \alpha-x_{C} \cos \alpha}{r_{b}}$.

Assuming the diameter of the column is $d_{R}$, and when $Z$ is an even number, the measuring distance of sprocket column is:

$$
M_{R}=\frac{m_{21} z \cos \alpha_{21}}{\cos \alpha_{B 1}}+d_{R}
$$

When $Z$ is an odd number, the measuring distance of sprocket column is:

$$
M_{R}=\frac{m_{21} z \cos \alpha_{21}}{\cos \alpha_{B 1}} \cos \frac{90^{\circ}}{z}+d_{R}
$$

$\alpha_{B 1}$ is the involute pressure angle when the involute tooth profile is through the centre of sprocket column,

$$
i n v \alpha_{B 1}=\frac{2 x \tan \alpha_{21}}{z}+i n v \alpha_{21}+\frac{d_{R}}{m_{2} z \cos \alpha_{21}}-\frac{\pi}{2 z} .
$$

Assuming the first tool normal pitch $P_{21}$ is equal to $P_{11}$ and the first tool normal tooth angle $\alpha_{21}$ is equal to $\alpha_{11}$, the modification coefficient $x_{1}$ corresponds to the tool $\left(P_{21}=P_{11}, \alpha_{21}=\alpha_{11}\right)$ can be obtained by a transformation formula which is:

$$
\frac{2 x_{1} \tan \alpha_{21}}{z}=i n v \alpha_{B 1}-i n v \alpha_{21}-\frac{d_{R}}{m_{21} z \cos \alpha_{21}}+\frac{\pi}{2 z} \text {. }
$$

Similarly, the second design system of dual phase Hy-Vo silent chain plate-sprocket -tool is established by using another dual phase sprocket pitch $P_{12}$, module $m_{12}$, pressure angle of sprocket $\alpha_{12}$.

\section{DESIGN EXAMPLE}

The basic pitch of dual phase Hy-Vo silent chain $P$ is $9.525 \mathrm{~mm}$; the hole pitch $A$ is $8.16 \mathrm{~mm}$; the big side surface curvature radius of the rocker-pin $r$ is 7.4 $\mathrm{mm}$; the distance between the circle centre $O$ of chain plate hole and the big side surface of the rocker-pin $S_{m}$ is $0.7 \mathrm{~mm}$; the positioning offset angle that rockerpin is locked in the chain plate $\gamma$ is $3.667^{\circ}$; the tooth profile angle of $\mathrm{Hy}-\mathrm{Vo}$ silent chain plate $\alpha$ is $30^{\circ}$; the benchmark apothem between the circle centre $O$, and the chain plate outside linear profile $f$ is $5 \mathrm{~mm}$.

According to the Eq. (1),

$$
P^{\prime}=A+2\left[\left(r+S_{m}\right) \cos \gamma-r\right]=9.5268 \mathrm{~mm} .
$$

Making $\delta=0.10 \sim 0.30 \mathrm{~mm}, R=93 \mathrm{~mm}, \lambda=1.6^{\circ}$, according to the Eq. (3), it could obtain:

$$
\left\{\begin{array}{l}
x_{1}=\frac{\left(R-f^{\prime}-\delta\right) \sin \left(\lambda+60^{\circ}\right)}{\cos \lambda}=78.287 \\
y_{1}=\frac{\left(R-f^{\prime}-\delta\right) \cos \left(\lambda+60^{\circ}\right)}{\cos \lambda}=42.33
\end{array} .\right.
$$

Making $P_{11}=P_{12}=P=9.523 \mathrm{~mm}, \alpha_{11}=\alpha_{12}=31.5^{\circ}$, $\sigma=\pi / z$.

When $Z=35,2 \theta=2 \pi / z$.

According to the Eq. (5),

$$
P^{\prime \prime}=A+2\left[\frac{r+S_{m} \cos (\theta-\gamma)-r}{\cos \theta}\right]=9.5603 \mathrm{~mm} .
$$

According to the Eq. (7),

$$
f_{01}=f_{2}-\left(\cot \frac{\pi}{z}-\sqrt{3}\right)\left(P^{\prime \prime}-P_{21}\right) / 4=3.966 \mathrm{~mm} \text {. }
$$

According to the Eq. (8), the modification coefficient $x$ corresponds to the hob $\left(P_{21}, \alpha_{21}\right)$ is:

$$
x=\frac{\pi \cot \alpha_{21}}{4}-\frac{z}{2}+\frac{\pi}{2 \tan (\pi / z)}-\frac{\pi f_{01}}{P_{21} \sin \alpha_{21}}=-1.344
$$

so the modification coefficient $x$ corresponds to the hob $\left(P_{22}, \alpha_{22}\right)$.

Making $d_{R}=5.225 \mathrm{~mm}$, according to the Eq. (13),

$$
M_{R}=\frac{m_{21} z \cos \alpha_{21}}{\cos \alpha_{B 1}} \cos \frac{90^{\circ}}{z}+d_{R}=103.239 \mathrm{~mm}
$$

according to Eq. (14), the modification coefficient $x_{1}$ corresponds to the tool $\left(P_{21}=P_{11}, \alpha_{21}=\alpha_{11}\right)$ can be got, $x_{1}=-1.531$, so the modification coefficient $x_{1}$ corresponds to the tool $\left(P_{22}=P_{12}, \alpha_{22}=\alpha_{12}\right)$.

When $Z=35, \quad x=-1.319, \quad M_{R}=109.456 \mathrm{~mm}$, $x_{1}=-1.523$. 


\section{THE FLUCTUATION ANALYSIS OF THE DUAL PHASE TRANSMISSION SYSTEM}

\subsection{Fluctuation Quantity Mathematical Analysis}

Based on the above design example, the dual phase Hy-Vo silent chain transmission system is built, as shown in Fig. 5. The transmission system is decomposed into two single-phase Hy-Vo silent chain transmission systems. Fig. 6 shows a single-phase Hy-Vo silent chain transmission system that the top of sprocket tooth is up, while Fig. 7 shows a single-phase Hy-Vo silent chain transmission system so that the slot of the sprocket tooth is up.

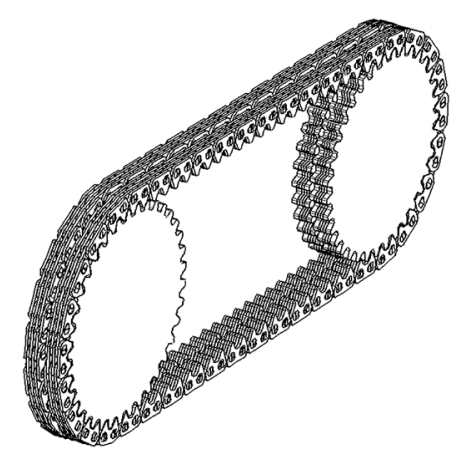

Fig. 5. The dual phase Hy-Vo silent chain transmission system

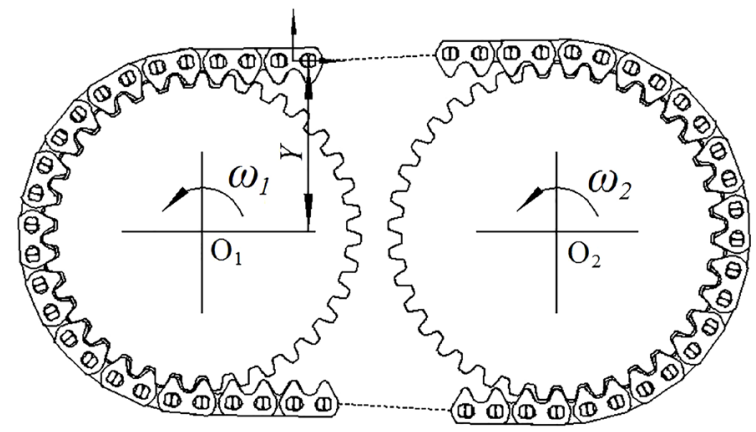

Fig. 6. Single-phase Hy-Vo silent chain transmission system that the top of the sprocket tooth is up

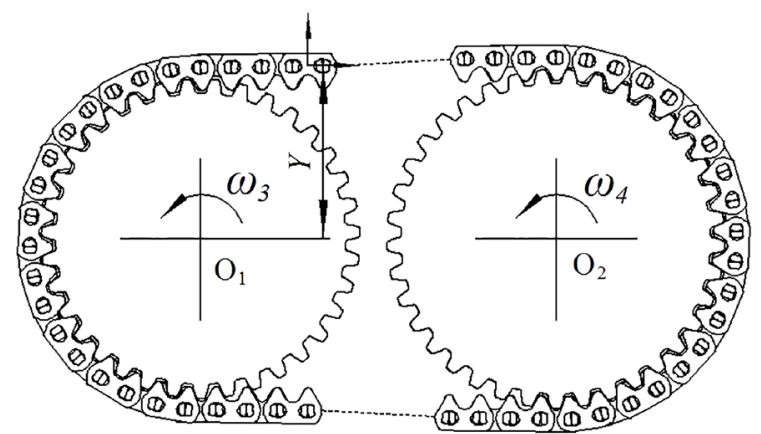

Fig. 7. Single-phase Hy-Vo silent chain transmission system that the slot of the sprocket tooth is up
The instantaneous transmission ratio in Fig. 6 is $i_{1}=\omega_{1} / \omega_{2}$, and the instantaneous transmission ratio in Fig. 7 is $i_{2}=\omega_{3} / \omega_{4}$. When two single-phase Hy-Vo silent chain transmission systems in Figs. 6 and 7 are engaging independently and simultaneously, at any time, $i_{1}$ is not necessarily equal to $i_{2}$, but in the dual phase transmission system, $i_{1}=i_{2}$. The instantaneous transmission ratio of dual phase transmission system may be different from the instantaneous transmission ratio of decomposed independently operating singlephase transmission systems, and the different instantaneous transmission ratio has a different effect on the tight-side centreline fluctuation quantity and the polygon effect; therefore, the tight-side centreline fluctuation quantity in the dual phase transmission system will be different from the fluctuation quantity in the independent operation single-phase transmission system .

The Fourier series of period $T=2 \pi$ about two hanging tight side fluctuations is established. Because $\sigma=\pi / z$, the Fourier series phase difference is $\pi$. It is assumed that fluctuation functions are:

$$
\left\{\begin{array}{c}
f_{1}=\sum_{1}^{\infty}\left[a_{n} \cos (k t)+b_{n} \sin (k t)\right] \\
f_{2}=\sum_{1}^{\infty}\left[a_{n} \cos (k t+\pi)+b_{n} \sin (k t-\pi)\right]
\end{array} .\right.
$$

Therefore, for the round pin silent chain system, the outer meshing Hy-Vo silent chain system and the inner-outer compound meshing Hy-Vo silent chain system, $f_{1}+f_{2}=0$, namely, the fluctuation quantity of two tight sides can be superimposed on a straight line in the dual phase Hy-Vo silent chain transmission system. For the new type Hy-Vo silent chain systems that include outer meshing $\mathrm{Hy}-\mathrm{Vo}$ silent chain and the inner-outer compound meshing $\mathrm{Hy}-\mathrm{Vo}$ silent chain, $f_{1}+f_{2} \approx 0$, namely, the fluctuation quantity of two tight side can be superimposed on a straight line approximately.

\subsection{Fluctuation Quantity Simulation Analysis}

The silent chain polygon effect has an important role in the stability of chain transmission process and the reduction of vibration noise. The tight-side centreline fluctuation quantity is the important indicator of the silent chain polygon effect. Based on the above design example, the new type Hy-Vo silent chain system model of the single-phase transmission is established, as shown in Fig. 8; so does the new type Hy-Vo silent chain system model of the dual phase transmission, as 
shown in Fig. 9. The tight-side centreline fluctuation quantity is extracted, and the silent chain polygon effect is studied.

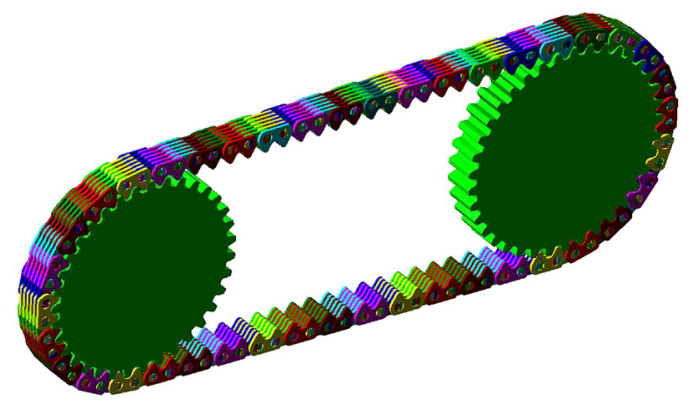

Fig. 8. The new type Hy-Vo silent chain system model of the singlephase transmission

The largest fluctuation quantity in a meshing period is extracted, Fig. 10 shows the largest fluctuation quantity about the single-phase transmission system. The horizontal axis is the time $t$, and the vertical axis $Y$ is the coordinate whose link plate centroid is in the vertical direction. The parameter $Y$ is illustrated in Figs. 6 and 7, and the maximum fluctuation quantity of the measuring link plate is $0.40 \mathrm{~mm}$.

Fig. 11 shows the largest fluctuation quantity about the dual phase transmission system and the maximum fluctuation quantity of the measuring link plate is $0.26 \mathrm{~mm}$. In comparison with the new type Hy-Vo silent chain system of the single-phase transmission, the largest fluctuation quantity about the new type Hy-Vo silent chain system of the dual phase transmission is reduced by 35 percent, and the polygon effect of dual phase transmission system is smaller.

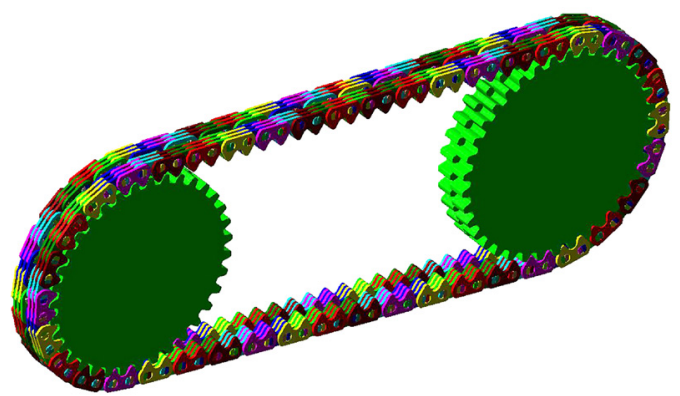

Fig. 9. The new type Hy-Vo silent chain system model of the dual phase transmission

\section{CONCLUSIONS}

(1) Based on the theory that the Hy-Vo silent chain plate profile is regarded as a tool profile of involute tooth, The meshing design system of

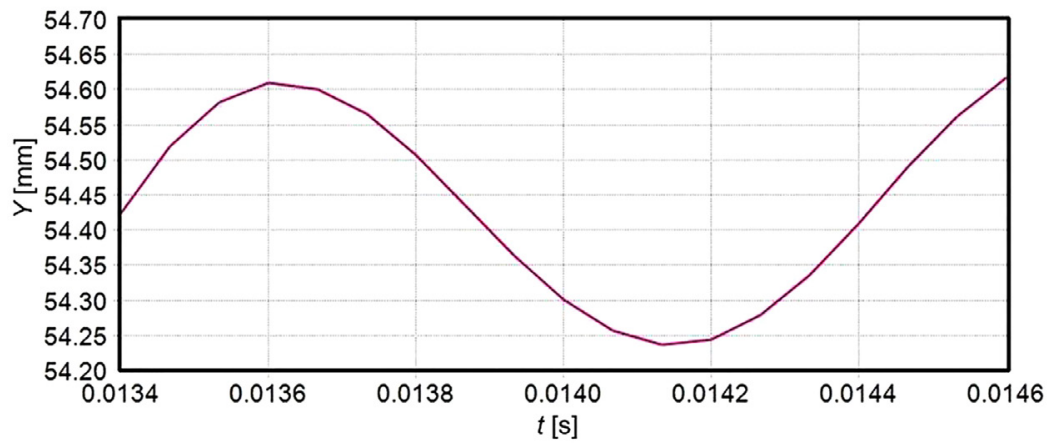

Fig. 10. The largest fluctuation quantity about the single-phase transmission system

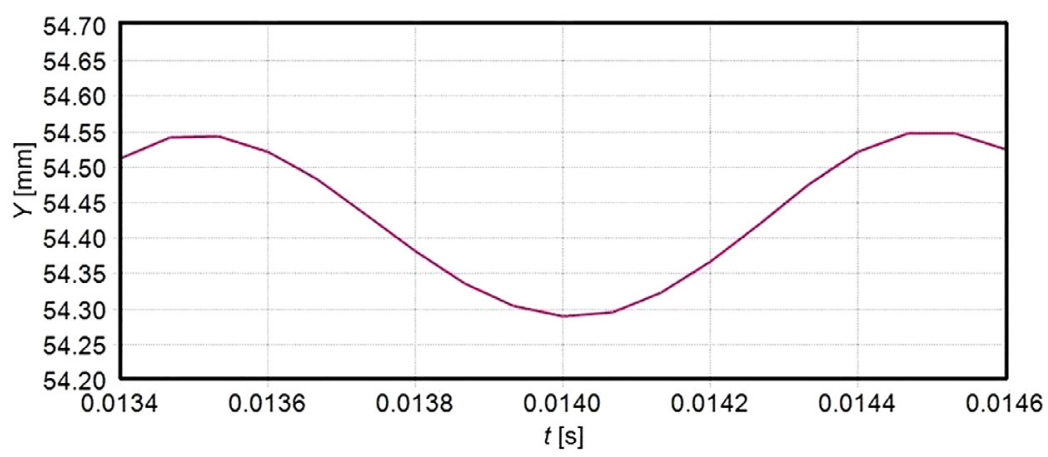

Fig. 11. The largest fluctuation quantity about the dual phase transmission system 
Hy-Vo silent chain-sprocket-tool is established. It is not only suitable for the design of the singlephase Hy-Vo silent chain transmission system, but also for the design of the dual phase Hy-Vo silent chain transmission system, the design method of the dual phase Hy-Vo silent chain plate-sprockettool is a new kind of design method.

(2) If $P_{11} \neq P_{12}$ or $\alpha_{11} \neq \alpha_{12}$, the two design system of the dual phase Hy-Vo silent chain plate- sprockettool will be different. If $P_{12} \neq P_{22}, \alpha_{12} \neq \alpha_{22}$, the design system of the dual phase Hy-Vo silent chain plate-sprocket-tool can be built through the relevant parameters transformation.

(3) When $P_{11}=P_{12}$ or $\alpha_{11}=\alpha_{12}$, the instantaneous transmission ratio of dual phase transmission system may be different from the instantaneous transmission ratio of decomposed independent operation single-phase transmission system. In comparison with the single-phase transmission system, the polygon effect of dual phase transmission system is smaller.

\section{ACKNOWLEDGEMENTS}

The research presented in this paper was supported by National Natural Science Foundation of China (No. 51305154).

\section{REFERENCES}

[1] Meng, F., Li, C., Cheng, Y. (2007). Proper conditions of meshing for Hy-Vo silent chain and sprocket. Chinese Journal of Mechanical Engineering, vol. 20, no. 4, p. 57-59. (in Chinese)

[2] Liu, X. Ding, S. Sun, W. Liu, J. Lu, J. Xu, M. (2011). Study on join fastness of silent chain. Proceedings of International Conference on Management Science and Intelligent Control, p. 275-278. (in Chinese)

[3] Meng, F., Feng, Z., Chu, Y. (2006). Meshing theory and design method of new silent chain and sprocket. Chinese Journal of Mechanical Engineering, vol. 19, no. 3, p. 425-427, DOI:10.3901/CJME.2006.03.425. (in Chinese)

[4] Meng, F., Liu, X., Xu, S. (2008). Meshing analysis and design of rotundity-datum-aperture Hy-Vo silent chain. Proceedings of the Institution of Mechanical Engineers, Part C: Journal of
Mechanical Engineering Science, vol. 222, no. 7, p. $1297-$ 1303, DOI:10.1243/09544062JMES865.

[5] Meng, F., Wang, S., Lan, H., Xu, R., Xu, H. (2005). Wear failure mechanism and multi-impact property of automotive engine chain. Chinese Journal of Mechanical Engineering, vol. 18, no. 3, p. 401-404, DOI:10.3901/CJME.2005.03.401. (in Chinese)

[6] Cheng, Y., Meng, F., Xu, H., Wu, J. (2007). Experimental study on high speed characteristics of automotive engine Oil-pump chain. Chinese Journal of Mechanical Engineering, vol. 20, no. 1, p. 36-39, Dol:10.3901/CJME.2007.01.036. (in Chinese)

[7] Wang, Y., Ji, D., Zhan, K., (2013). Modified sprocket tooth profile of roller chain drives. Mechanism and Machine Theory, vol. 70, p. 380-393, D0I:10.1016/j.mechmachtheory.2013.08.006.

[8] Liu, X., Wang, W., Sun, W., Wu, T., Liu, J.., Liu, J.. (2012). Design and experimental analyse of low noise double-pitch silent chain for conveyor. Procedia Engineering, vol. 29, p. 21462150, D0I:10.1016/j.proeng.2012.01.277.

[9] Sun, W., Liu, X., Liu, J. (2012). Experimental investigation of sprocket tooth form effect on dynamic tension of silent chain. Research Journal of Applied Sciences, Engineering and Technology, vol. 4, no. 7, p. 846-850.

[10] Bucknor, N.K., Freudenstein, F. (1994). Kinematic and static force analysis of rocker-pin jointed silent chains with involute sprockets. ASME Journal of Mechanical Design, vol. 116, no. 3, p. 842-848, Dol:10.1115/1.2919458.

[11] Troedsson, I., Vedmar, L. (2001). A dynamic analysis of the oscillations in a chain drive. ASME Journal of Mechanical Design, vol. 123, no. 3, p. 395-401, Dol:10.1115/1.1374196.

[12] Stephenson, R., Glennie, D., Fawcett, J.N., Hale, J.M. (2000). A method of measuring the dynamic loads in high-speed timing chains. Proceedings of the Institution of Mechanical Engineers, Part D: Journal of Automobile Engineering, vol. 214, no. 2, p. 217-226, Dol:10.1177/095440700021400211.

[13] Schwab, A.L., Meijaard, J.P., Meijers, P. (2002). A comparison of revolute joint clearance models in the dynamic analysis of rigid and elastic mechanical systems. Mechanism and Machine Theory, vol. 37, no. 9, p. 895-913, Dol:10.1016/ S0094-114X(02)00033-2.

[14] Flores, P., Ambrósio, J., Claro, J.C.P., Lankarani, H.M., Koshy, C.S. (2009). Lubricated revolute joints in rigid multibody systems. Nonlinear Dynamics, vol. 56, no. 3, p. 277-295, DOI:10.1007/s11071-008-9399-2.

[15] Pereira, C.M., Ambrósio, J.A., Ramalho, A.L. (2010). A methodology for the generation of planar models for multibody chain drives. Multibody System Dynamics, vol. 24, no. 3, p. 303-324, D0l:10.1007/s11044-010-9207-x. 